\title{
Cytopathological Features of a Severe Type of Corneal Intraepithelial Neoplasia
}

\author{
Hideki Fukuoka ${ }^{a, b}$ Satoshi Kawasaki ${ }^{c, d}$ Norihiko Yokoi ${ }^{d}$ \\ Kenta Yamasaki ${ }^{d}$ Shigeru Kinoshita ${ }^{d}$ \\ ${ }^{a}$ Division of Ophthalmology, Department of Advanced Medicine, National Center for \\ Geriatrics and Gerontology, Obu, Japan; ${ }^{b}$ Shiley Eye Institute, University of California, \\ San Diego, Calif., USA; 'Department of Ophthalmology, Osaka University Graduate School \\ of Medicine, Osaka, Japan; ${ }^{d}$ Department of Ophthalmology, Kyoto Prefectural University \\ of Medicine, Kyoto, Japan
}

\section{Keywords}

Corneal intraepithelial neoplasia - Cell proliferation · Apoptosis - Epithelial mesenchymal transition $\cdot$ Cytokeratin expression pattern $\cdot$ Keratinization

\begin{abstract}
Purpose: To report the cytopathological features of corneal intraepithelial neoplasia (CIN) through the investigation of cytokeratin expression pattern, keratinization, cell proliferation, apoptosis, and epithelial mesenchymal transition. Patient and Methods: Corneal tissue excised from a CIN patient was examined in this study. Cryosections of the excised CIN epithelial tissue were examined by immunostaining analysis using antibodies against cytokeratins, keratinization-related proteins, Ki-67, human telomerase reverse transcriptase (hTERT), and epithelial mesenchymal transition (EMT)-related proteins. Subcellular localization of F-actin was also analyzed using phalloidin. For the detection of apoptotic cells, terminal deoxynucleotidyl transferase dUTP nick end labeling (TUNEL) assay was performed. Real-time polymerase chain reaction was performed to quantify the expression level of hTERT in the CIN epithelium. Results: The CIN epithelium exhibited a significantly altered cytokeratin expression pattern compared to normal corneas with an upregulated expression of keratinizationrelated proteins. The CIN epithelium also demonstrated an increased number of Ki-67-
\end{abstract}


Case Reports in
Ophthalmology

Case Rep Ophthalmol 2016;7:253-261 $10.1159 / 000445937$

(C) 2016 The Author(s). Published by S. Karger AG, Basel www.karger.com/cop

Fukuoka et al.: Cytopathological Features of a Severe Type of Corneal Intraepithelia Neoplasia

positive cells with an upregulated expression of hTERT, while exhibiting an increased number of apoptotic cells. EMT did not occur in the CIN epithelium. Conclusion: CIN epithelium seems to be slightly dedifferentiated from the corneal epithelial lineage. The status of cell proliferation and apoptosis in the CIN epithelium was significantly altered from that of normal corneal epithelium, but its malignancy level does not appear to be as high as that of metastasis-competent malignant cancers.

\section{Introduction}

Intraepithelial neoplasia, a condition in which cancer cells are limited to the epithelial layer, can occur both in corneal and conjunctival ocular surface epithelium. Corneal intraepithelial neoplasia (CIN) was first described in 1984 by Waring et al. [1], and it can reportedly affect patients of all ages, but predominantly occurs in older males with an average age of 56 years. Several risk factors have been reported for this disease, including chronic ultraviolet exposure, chemical exposure, contact lens wear, human papillomavirus, and smoking [2]. Typically, CIN lesions are slightly elevated with a pearly gray appearance, are well demarcated from the normal region of the epithelial surface, and frequently have characteristic engorged vessels.

The purpose of this study was to investigate the cytokeratin expression patterns, keratinization, cell proliferation, apoptosis, and epithelial-mesenchymal transition (EMT) of CIN tissue in order to elucidate the cytological nature of this disease.

\section{Materials and Methods}

\section{Normal Corneal and CIN Tissue Preparation}

CIN tissue was obtained from a CIN patient at the time of surgery. Normal corneal tissues were obtained from the North West Lions Eye Bank (Seattle, Wash., USA). Both the CIN tissues and the normal tissues were embedded (Tissue-Tek OCT; Sakura Fine Technical Co., Ltd., Tokyo, Japan), snap frozen with liquid nitrogen, and stored at $-80^{\circ} \mathrm{C}$.

\section{Immunostaining Analysis}

The 8- $\mu \mathrm{m}$-thick tissue sections were placed on glass slides for immunostaining analysis. The sections were dried and then fixed with Zamboni's fixative. After washing with $0.01 \mathrm{M}$ phosphate-buffered saline, the sections were incubated with blocking solution containing $1 \%$ bovine serum albumin in $0.01 \mathrm{M}$ phosphate-buffered saline. Then, the sections were incubated with primary antibodies or phalloidin (table 1) as well as their corresponding isotype controls. After washing, the sections were incubated with fluorescence-labeled secondary antibodies (Alexa Fluor 488; Life Technologies Corp., Carlsbad, Calif., USA). After washing again, the sections were covered with coverslips, and then photographed by use of a fluorescent microscope (AX70 TRF; Olympus Corp., Tokyo, Japan).

\section{Terminal Deoxynucleotidyl Transferase dUTP Nick End Labeling Assay}

Terminal deoxynucleotidyl transferase dUTP nick end labeling (TUNEL) assay was performed using a commercially available kit (DeadEnd ${ }^{\mathrm{TM}}$ Fluorometric TUNEL System; Promega Corp., Madison, Wis., USA). Briefly, the 8- $\mu$ m-thick cryosections were dried and permeabilized with $0.2 \%$ Triton X-100 solution, and then incubated in a buffer containing 


\section{Case Reports in Ophthalmology}

\begin{tabular}{|l|l|}
\hline $10.1159 / 000445937$ & (c) 2016 The Author(s). Published by S. Karger AG, Basel \\
\hline
\end{tabular} www.karger.com/cop

Fukuoka et al:: Cytopathological Features of a Severe Type of Corneal Intraepithelia Neoplasia

recombinant terminal deoxynucleotidyl transferase and fluorescein-12-dUTP to detect $3^{\prime}$ ends of fragmented genomic DNA. After washing, the sections were counterstained with propidium iodide solution, covered with coverslips, and photographed.

\section{RNA Extraction, cDNA Synthesis, and Quantitative Polymerase Chain Reaction}

RNA was extracted using the RNeasy ${ }^{\circledR}$ Micro Kit (QIAGEN GmbH, Hilden, Germany). Briefly, epithelial cells of the CIN tissue were lysed in a lysis buffer and applied onto a silicabased column. After DNase I treatment, yield and quality checking were done by electrophoresis using a capillary-based bioanalyzer (Agilent 2100 Bioanalyzer; Agilent Technologies, Inc., Santa Clara, Calif., USA). The RNA samples were then reverse-transcribed by use of the Transcriptor First Strand cDNA Synthesis Kit (Roche Diagnostics GmbH, Mannheim, Germany). Quantitative polymerase chain reaction (q-PCR) was performed using the ABI Prism 7000 Sequence Detection System (Applied Biosystems Japan Ltd., Tokyo, Japan). Briefly, cDNA samples were amplified using $10 \mathrm{pmol} / \mathrm{l}$ of primer pairs designed for human telomerase reverse transcriptase (hTERT; hTERT_F: ACGGCGACATGGAGAACAA, hTERT_R: CACTGTCTTCCGCAAGTTCAC) and glyceraldehyde 3-phosphate dehydrogenase (GAPDH; GAPDH_F: GAAGGTGAAGGTCGGAGT, GAPDH_R: GAAGATGGTGATGGGATTTC). The relative expression of the hTERT gene in each sample was calculated by the $\triangle \triangle \mathrm{CT}$ method using the GAPDH gene as an internal control.

\section{Case Report}

An otherwise healthy 87-year-old male presented with complaints of foreign body sensation and tearing in his left eye. Our initial diagnosis was recurrent corneal erosion due to severe superficial punctate keratopathies, and epithelial microcysts were found at the central region of his left cornea. After several regular checkups, a grayish, slightly thickened epithelial lesion had become apparent at the upper temporal side of the corneal limbus in his left eye. The patient underwent an initial operation to excise the total epithelium of the abnormal region. During the course of several, regular postoperative checkups, a dysplastic lesion recurred, and then gradually enlarged until the lesion covered almost the entire cornea and $360^{\circ}$ of the corneal limbus (fig. 1). The lesion appeared to be supported by adjacent engorged vessels. At that point, visual acuity of his left eye was 0.5 (decimal visual acuity), and the intraocular pressure of his left eye was normal. A total resection of the abnormal lesion was then performed. Since this 2 nd operation removed virtually all of the limbal epithelial cells which potentially harbor corneal epithelial stem cells, allogeneic keratoepithelioplasty was also performed to prevent conjunctivalization of the cornea. Moieties of the resected tissues at the 2 nd operation were subjected to routine pathological examination, and the resultant diagnosis was grade III CIN with significantly thickened epithelium, an increased amount of mitosis, and abnormal cellular polarity (stage Tis).

Using the tissue resected at the 2nd operation, histological examinations aimed at investigating cytokeratins were performed. Cytokeratin 1 was expressed in a patchy fashion in the suprabasal to upper layers of the CIN epithelium, but not expressed in the normal corneal samples. Cytokeratin 3 was diffusely expressed in the suprabasal to upper layers of the CIN epithelium, while all epithelial layers diffusely expressed this cytokeratin in the normal corneal samples (fig. 2). Cytokeratin 4 was expressed in a patchy fashion in the middle to upper layers of the CIN epithelium, while the normal corneal samples expressed this cytokeratin only in the superficial epithelial layer. Cytokeratin 6 was diffusely expressed in the middle to 


\section{Case Reports in Ophthalmology} \begin{tabular}{|l|l}
\hline $10.1159 / 000445937$ & $\begin{array}{l}\text { C } 2016 \text { The Author(s). Published by S. Karger AG, Basel } \\
\text { www.karger.com/cop }\end{array}$ \\
\hline
\end{tabular}

Fukuoka et al:: Cytopathological Features of a Severe Type of Corneal Intraepithelia Neoplasia

upper layers of the CIN epithelium, while the normal corneal samples exhibited virtually no expression of this cytokeratin in any of the epithelial layers. Cytokeratin 8 was not expressed in any of the epithelial layers of both the CIN and the normal corneal samples. Cytokeratin 12 was expressed in a patchy fashion in the suprabasal to upper layers of the CIN epithelium, while the normal corneal samples expressed this cytokeratin in almost all epithelial layers at a much higher level. Cytokeratin 13 was expressed diffusely in the suprabasal to upper layers of the CIN epithelium, but was not expressed in any epithelial layers of the normal corneal samples. Cytokeratin 16 was expressed in a patchy fashion in the suprabasal to upper layers of the CIN epithelium, but was virtually not expressed in any epithelial layer of the normal corneal samples. For the assessment of keratinization, the expression of keratinization-related protein genes was investigated. Transglutaminase 1 and involucrin were expressed in almost all layers of the CIN epithelium as compared to the normal corneal samples. Filaggrin was not expressed in either the CIN samples or the normal corneal samples. Nuclei of all the superficial cells of the CIN epithelium remained as a shrunken pyknotic structure. Some of the immunostaining results against the cytokeratins and keratinizationrelated proteins were confirmed by q-PCR, with the obtained data being mostly compatible (data not shown).

A large number of Ki-67-positive cells were localized around the basal to middle layers of the CIN epithelium, while only a limited number of Ki-67-positive cells were found in the basal to suprabasal layers of the normal corneal samples. TUNEL-positive cells were mainly detected in the middle to upper layers of the CIN tissue, while virtually no TUNEL-positive cells were detected in the normal corneal samples. The CIN epithelium expressed hTERT protein in almost all layers, while virtually no expression of this protein was found in the normal corneal samples. Epithelial cells of the CIN tissue exhibited the significantly upregulated expression of hTERT mRNA compared to those of the normal corneal samples. The expression level was comparable to that measured in the human corneal epithelial cells, the human corneal epithelial large $\mathrm{T}$ antigen cells, a cell line which was immortalized by the adenoviral introduction of SV40 large T antigen (fig. 3).

We found that the CIN epithelium strongly expressed the epithelial-specific marker Ecadherin, but did not express the mesenchymal marker $\alpha$-smooth muscle actin. In addition, the CIN epithelium did not express the other mesenchymal marker, $\mathrm{N}$-cadherin (fig. 3), while the normal limbal epithelium exhibited the expression of this protein at its basal layer, where stem cells of the corneal epithelial cells reside. We also found that filamentous actin (F-actin) was accumulated mainly near the cell-to-cell boundaries of the CIN epithelial cells, which means that the CIN epithelium has an epithelial phenotype.

\section{Discussion}

In this study, we investigated cytokeratin expression patterns, statuses of cell proliferation, apoptosis, and EMT to assess the cellular properties of CIN epithelium. Cytokeratins 3 and 12 were expressed in the CIN tissue, although their expression level was much lower than that of the normal corneal samples. Because these cytokeratins are specifically expressed in normal corneal epithelium [3], it is suggested that CIN epithelium partially loses the nature of corneal epithelium during the course of malignant transformation. Meanwhile, cytokeratins 4 and 13, which are expressed mainly in the stratified mucous epithelia such as conjunctiva [3], were found to be upregulated in the CIN tissue. In addition, cytokeratin 1, which is expressed only in the epidermis [3], was found to be expressed in the CIN epitheli- 


\section{Case Reports in Ophthalmology}

um. The CIN epithelium did not express cytokeratin 8, which is a marker for simple epithelia such as colon epithelium [3], suggesting that the CIN epithelium did not acquire the cellular properties of simple epithelia. These results suggest that the CIN epithelium slightly loses its original cell features, but acquires those properties of non-corneal-type stratified wet epithelium (e.g., conjunctival epithelium) including epidermal epithelium as well as dry epithelium (epidermis).

In the normal keratinization process of skin epidermis, filaggrin serves as a matrix for the aggregation of cytokeratin $1 / 10$ intermediate filaments to establish microfibrils at the cornified layer [4]. The catalytic enzyme of transglutaminase 1 cross-links involucrin, to form cornified cell envelopes in the lower part of the cornified layer [5]. Our results revealed that most of these molecules, except for filaggrin, were expressed in the CIN epithelium. However, the keratinization process that possibly occurred in the CIN epithelium appears not to be a complete type of keratinization, but an incomplete-type keratinization, because nuclei with a shrunken pyknotic structure still remained in the superficial cells of the CIN epithelium. Since complete-type keratinization is sometimes found in severe cases of Stevens-Johnson syndrome [6], it is speculated that the keratinization stimuli that occurred in the CIN tissue was not so strong. Several previous reports have demonstrated the existence of keratinization in tumors derived from non-keratinized epithelial tissues such as those of the breast [7], esophagus [8], and lung [9]. Therefore, keratinization seems to be a common phenomenon in many types of epithelium-derived tumors. It is speculated that the CIN epithelium was in a well-differentiated status since keratinization is generally thought to be a marker of well-differentiated tumors.

Cytokeratins 6 and 16, which are markers of hyperproliferation [10], were highly expressed in the CIN epithelium, while the normal corneal samples did not express these cytokeratins. In addition, Ki-67, a cell proliferation marker, was detected in a large number of cells located at the basal to middle layers of the CIN epithelium, while only a limited number of Ki-67-positive cells were found in the normal corneal samples. These results indicate that the CIN epithelium was in an abnormally hyperproliferative status, which is in good agreement with the results of the pathology examination.

It is generally accepted that acquirement of an invasive potential into surrounding tissues requires EMT [10]. In this study, the CIN epithelium demonstrated expression of Ecadherin, but was devoid of $\alpha$-smooth muscle actin as well as $\mathrm{N}$-cadherin. These results were almost identical to those of the normal corneal epithelia, suggesting that there was no occurrence of EMT in the CIN epithelium. Therefore, these findings strongly suggest that the CIN epithelium did not have metastatic and invasive potentials, which indicates that the prognosis of the CIN epithelium seems good. This is concordant to the former results of apoptosis and keratinization, but discrepant to the results of cell proliferation and hTERT expression.

In summary, we investigated the cytopathological features of a case with CIN. We feel that the CIN epithelium is slightly dedifferentiated in the aspect of the cytokeratin expression pattern. As for cancer properties, the CIN epithelium has two apparently contradictory sides: one is upregulated cell proliferation with increased hTERT expression, and the other side is the existence of keratinization, increased apoptosis, and absence of EMT. From these somewhat conflicting results, we feel that the CIN's malignancy level is not as high as the level in which invasion or distant metastasis occurs. Such cellular features seem to be closely associated with the pathological findings that CIN patients are generally devoid of any distant metastasis. We hope this current study will contribute to future investigations aimed at a better understanding of this disease, as well as the development of better treatments. 


\section{Acknowledgements}

This work was supported by a grant-in-aid (No. 20390451) from the Japanese Ministry of Education, Science, Culture and Sports, and a grant from the Japanese Ministry of Health, Labour and Welfare. This work was also supported by a research fund from the Kyoto Foundation for the Promotion of Medical Science. The authors wish to thank John Bush for reviewing the manuscript.

\section{Statement of Ethics}

This study was conducted with adherence to the tenets of the Declaration of Helsinki. This research was approved by the committee for the ethical issues on human research of Kyoto Prefectural University of Medicine. Prior informed consent was obtained from the patient after receiving a complete explanation of the nature and possible consequences of the study.

\section{Disclosure Statement}

The authors report no conflicts of interest in this work.

\section{References}

1 Waring GO, Roth AM, Ekins MB: Clinical and pathologic description of 17 cases of corneal intraepithelial neoplasia. Am J Ophthalmol 1984;97:547-559.

-2 Auw-Haedrich C, Sundmacher R, Freudenberg N, Spelsberg H, Feltgen N, Maier P, et al: Expression of p63 in conjunctival intraepithelial neoplasia and squamous cell carcinoma. Graefes Arch Clin Exp Ophthalmol 2006;244:96-103.

-3 Moll R, Franke WW, Schiller DL, Geiger B, Krepler R: The catalog of human cytokeratins: patterns of expression in normal epithelia, tumors and cultured cells. Cell 1982;31:11-24.

-4 Wu R-L, Galvin S, Wu S-K, Xu C, Blumenberg M, Sun T-T: A 300 bp 5'-upstream sequence of a differentiation-dependent rabbit K3 keratin gene can serve as a keratinocyte-specific promoter. J Cell Sci 1993;105:303-316.

5 Rice RH, Green H: Presence in human epidermal cells of a soluble protein precursor of the cross-linked envelope: activation of the cross-linking by calcium ions. Cell 1979;18:681-694.

6 Nakamura T, Nishida K, Dota A, Matsuki M, Yamanishi K, Kinoshita S: Elevated expression of transglutaminase 1 and keratinization-related proteins in conjunctiva in severe ocular surface disease. Invest Ophthalmol Vis Sci 2001;42:549-556.

7 Hennessy BT, Krishnamurthy S, Giordano S, Buchholz TA, Kau SW, Duan Z, et al: Squamous cell carcinoma of the breast. J Clin Oncol 2005;23:7827-7835.

-8 Sarbia M, Bittinger F, Porschen R, Dutkowski P, Willers R, Gabbert HE: Prognostic value of histopathologic parameters of esophageal squamous cell carcinoma. Cancer 1995;76:922-927.

-9 Kogan EA, Ugriumov DA, Jaques G: Morphologic and molecular-genetic characteristics of keratinization and apoptosis in squamous cell lung carcinoma. Arkh Patol 2000;62:16-20.

10 Thiery JP: Epithelial-mesenchymal transitions in tumour progression. Nat Rev Cancer 2002;2:442-454. 


\section{Case Reports in Ophthalmology}

\begin{tabular}{l|l}
\hline Case Rep Ophthalmol 2016;7:253-261 \\
\hline $10.1159 / 000445937$ & $\begin{array}{l}\text { C } 2016 \text { The Author(s). Published by S. Karger AG, Basel } \\
\text { www.karger.com/cop }\end{array}$ \\
\hline
\end{tabular}

Fukuoka et al:: Cytopathological Features of a Severe Type of Corneal Intraepithelia Neoplasia
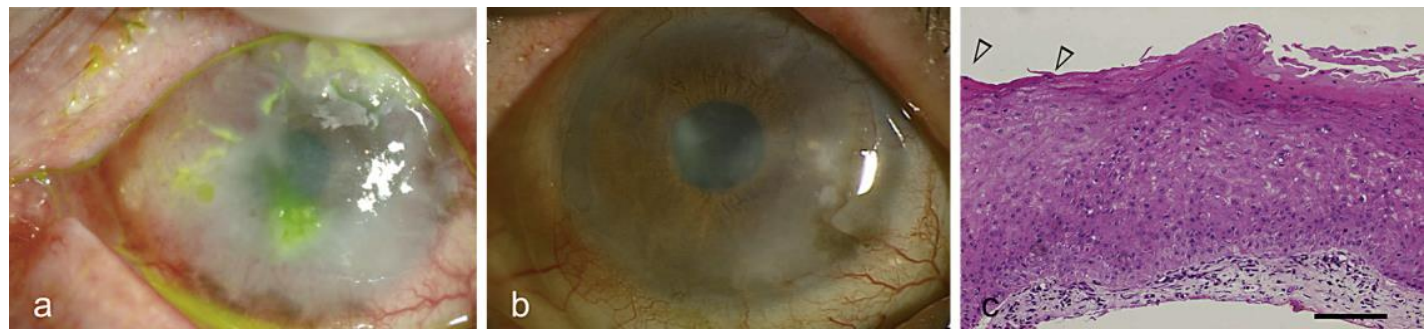

Fig. 1. Slit-lamp images of the left eye of the CIN patient before the 2 nd operation (a) and 14 months after the 2nd operation (b). Histological examination of the CIN tissue (c). Arrow heads indicate that nuclei of the superficial cells of the CIN epithelium remained as a shrunken pyknotic structure. H\&E staining; bar = $100 \mu \mathrm{m}$.

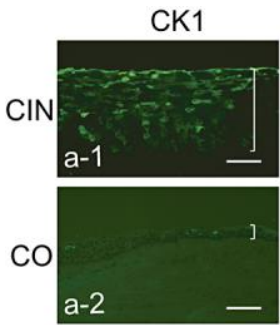

CK12

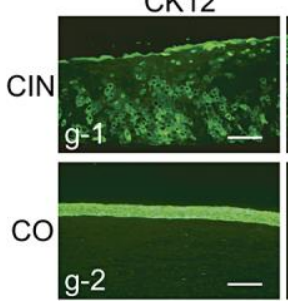

CK3
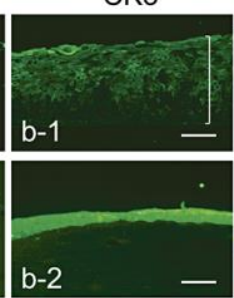

CK13

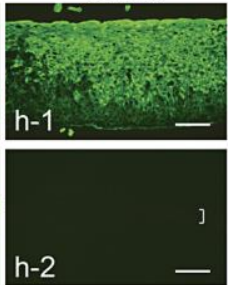

CK4
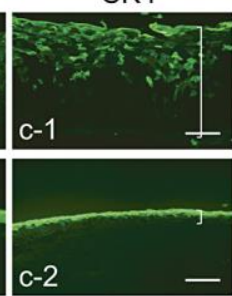

CK16

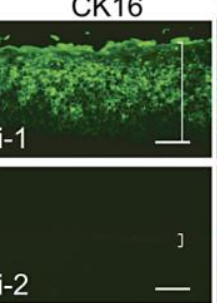

CK6

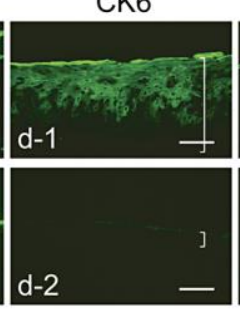

Transglutaminase

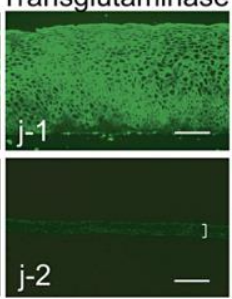

CK8
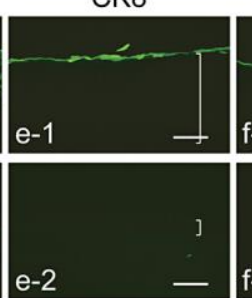

Involucrin

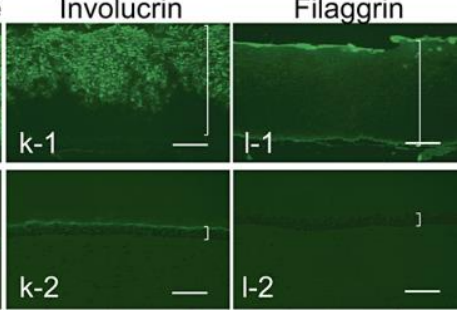

Fig. 2. Images showing immunostaining results against cytokeratins and keratinization markers. Cytokeratins 1 (a-1, a-2), 3 (b-1, b-2), 4 (c-1, c-2), 6 (d-1, d-2), 8 (e-1, e-2), 10 (f-1, f-2), 12 (g-1, g-2), 13 (h-1, $\mathbf{h}-2$ ), and 16 (i-1, i-2), and transglutaminase (j-1, j-2), involucrin (k-1, k-2), and filaggrin (I) for the CIN tissue $(\mathbf{a}-\mathbf{1 - k}-\mathbf{1})$ and the normal corneal tissue $(\mathbf{a}-\mathbf{2}-\mathbf{k}-\mathbf{2})$. Bar $=100 \mu \mathrm{m}$. The brackets indicate the CIN and corneal epithelial layer. Note that the superficial staining seen in the CIN epithelium is an artifact, because it was also found in the negative control samples. 

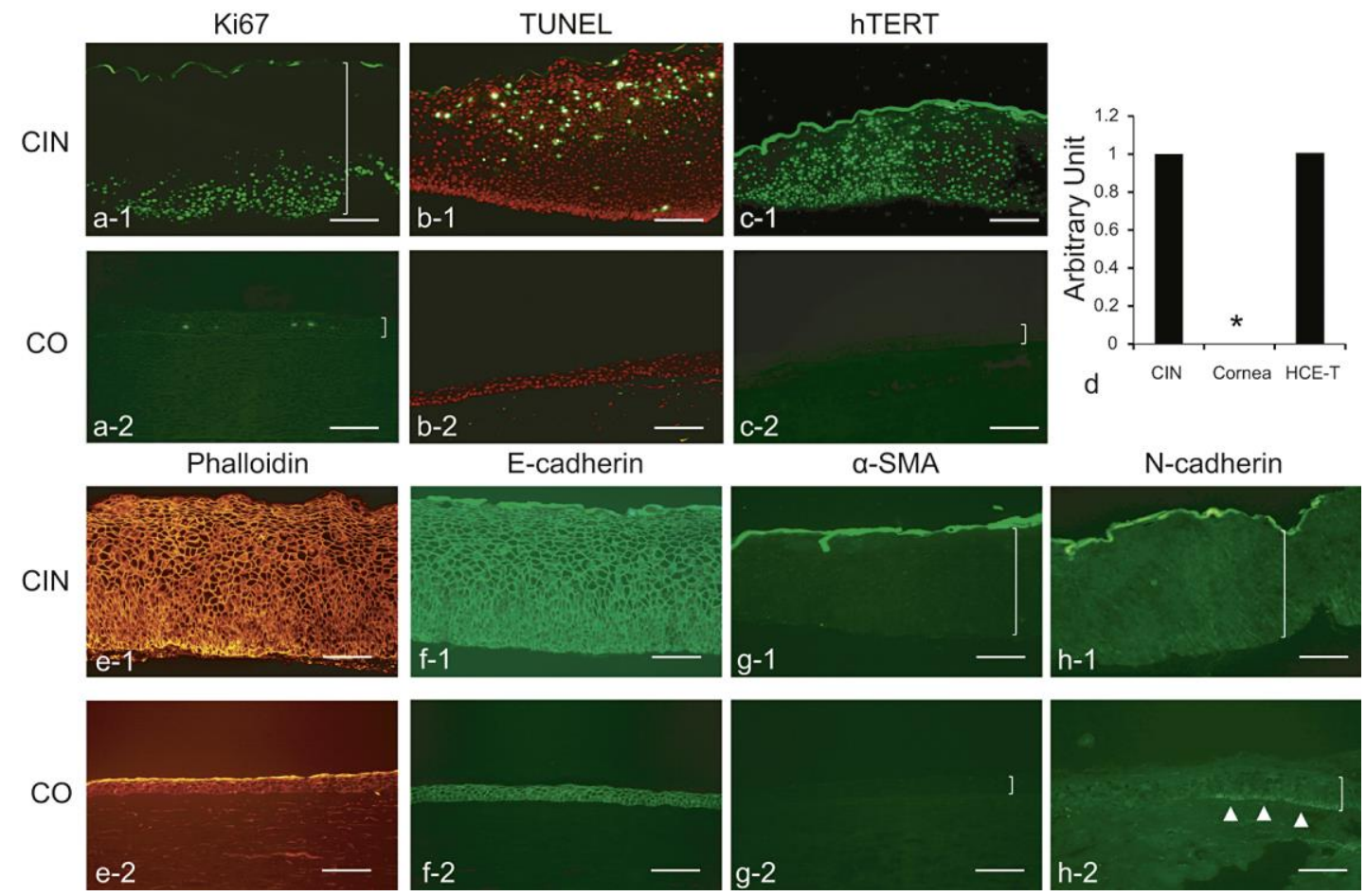

Fig. 3. Images of the immunostaining results. Immunostaining against Ki-67 (a-1, a-2), the results of TUNEL analyses (b-1, b-2), hTERT expression (c-1, c-2), phalloidin (e-1, e-2) and antibodies against E-cadherin (f-1, $\mathbf{f - 2}), \alpha$-smooth muscle actin $(\alpha \mathrm{SMA})(\mathbf{g}-\mathbf{1}, \mathbf{g}-\mathbf{2})$, and N-cadherin $(\mathbf{h}-\mathbf{1}, \mathbf{h}-\mathbf{2})$ for the CIN tissue (a-1-c-1, e-1-h-1) and the normal corneal tissue (a-2-c-2, e-2-h-2). Bar $=100 \mu \mathrm{m}$. d Real-time PCR data about the expression of hTERT mRNA. The asterisk means that the signal was below the detection limit. The arrow heads indicate cells positive for $\mathrm{N}$-cadherin, which are thought to be potential corneal epithelial stem cells. HCE-T = Human corneal epithelial large T antigen cells. The CIN epithelium demonstrated a large number of apoptotic cells. The hTERT gene was significantly upregulated in the CIN epithelium in both RNA and protein levels compared to the normal corneal epithelia. 
Table 1. Antibodies used in this study

\begin{tabular}{|c|c|c|c|c|c|}
\hline Antibody & $\begin{array}{l}\text { Type of } \\
\text { antibody }\end{array}$ & $\begin{array}{l}\text { Raising } \\
\text { animal }\end{array}$ & Clone & Source & Dilution \\
\hline Cytokeratin 1 & mono & mouse & $34 \mathrm{~B} 4$ & Novocastra & $\times 40$ \\
\hline Cytokeratin 3 & mono & mouse & AE5 & Santa Cruz & $\times 100$ \\
\hline Cytokeratin 4 & mono & mouse & 6B10 & Novocastra & $\times 200$ \\
\hline Cytokeratin 6 & mono & mouse & LHK-6B & Novocastra & $\times 200$ \\
\hline Cytokeratin 8 & mono & mouse & TS1 & Novocastra & $\times 400$ \\
\hline Cytokeratin 10 & mono & mouse & LHP1 & Novocastra & $\times 100$ \\
\hline Cytokeratin 12 & poly & goat & N16 & Santa Cruz & $\times 100$ \\
\hline Cytokeratin 13 & mono & mouse & KS-1A3 & Novocastra & $\times 200$ \\
\hline Cytokeratin 16 & mono & mouse & LL025 & Novocastra & $\times 40$ \\
\hline Transglutaminase 1 & mono & mouse & B.C1 & Biogenesis & $\times 20$ \\
\hline Involucurin & mono & mouse & SY5 & Novocastra & $\times 200$ \\
\hline Filaggrin & mono & mouse & 576 & Biogenesis & $\times 500$ \\
\hline hTERT & mono & mouse & $44 \mathrm{~F} 12$ & Novocastra & $\times 40$ \\
\hline Ki-67 & mono & mouse & Ki-S5 & Chemicon & $\times 40$ \\
\hline E-cadherin & mono & rat & ECCD-2 & TaKaRa & $\times 50$ \\
\hline Smooth muscle actin & mono & mouse & $1 \mathrm{~A} 4$ & DAKO & $\times 50$ \\
\hline N-cadherin & poly & rabbit & H63 & Santa Cruz & $\times 20$ \\
\hline Phalloidin & NA & NA & NA & MOP & $\times 400$ \\
\hline
\end{tabular}

